\title{
Diversidad del orden Lepidoptera (Hesperioidea y Papilionoidea) de la ciudad Corrientes, Argentina
}

\author{
María Gabriela Lazzeri ${ }^{1 *}$, María Esther $\operatorname{Bar}^{1} \&$ Miryam Pieri Damborsky ${ }^{1}$ \\ 1. Cátedra de Biología de los Artrópodos, Departamento de Biología, Facultad de Ciencias Exactas y Naturales y \\ Agrimensura, Universidad Nacional del Nordeste, Av. Libertad 5470, W 3404 AAS, Corrientes, Argentina, Tel: \\ 03783- 457950- Int. 419, Fax: 03783-473930-303; gabrielalazzeri@exa.unne.edu.ar, mebar@exa.unne.edu.ar, \\ mdambor@exa.unne.edu.ar \\ * Autor para correspondencia: gabidegabis@hotmail.com
}

Recibido 20-I-2010. C Corregido 30-VII-2010. Aceptado 31-VIII-2010.

\begin{abstract}
Diversity of the order Lepidoptera (Hesperioidea and Papilionoidea) from Corrientes city, Argentina. Urbanization is one of the most important threats for biodiversity. Among many different organisms, butterflies are useful indicators of environment diversity and quality. The aim of this study was to investigate the Lepidoptera from Corrientes city. Random samplings were performed at two sites: a native forest situated in Santa Catalina district and an urban area, Parque Mitre. The captures were carried out using entomological nets, at four seasons between January to October 2007. A total of 1114 butterflies, represented by six families: Hesperiidae, Lycaenidae, Nymphalidae, Papilionidae, Pieridae and Riodinidae and 18 subfamilies were recorded. Fifty-nine genera and 75 species were identified; Anartia jatrophae jatrophae was the most abundant species at both localities. This species and Urbanus procne, Phoebis sennae marcellina, Pyrgus orcus and Dryas iulia alcionea were, among other seven, captured at all months. Highest values of abundance were registered during the warmest seasons. Santa Catalina presented the largest abundance $(n=701)$, richness $(S=74)$ and diversity $\left(H^{\prime}=3.87\right)$. A total of 413 individuals and 52 species were identified at Parque Mitre, and Shannon diversity index was 3.58. The obtained data reveals a high species richness and similarity at both sites. Rev. Biol. Trop. 59 (1): 299-308. Epub 2011 March 01.
\end{abstract}

Key words: diurnal Lepidoptera, Hesperioidea, Papilionoidea, species richness, seasonality, urban park, suburban area.

El orden Lepidoptera es uno de los más diversos entre los insectos y se destaca por su abundancia en América del Sur (Fraija \& Fajardo 2006). Se estima en más de 250000 las especies identificadas, de las cuales unas 150000 han sido descritas hasta el presente. La Región Neotropical exhibe la mayor diversidad de Lepidoptera, alberga el $42 \%$ del total de especies, cuando son tenidas en cuenta sólo Papilionoidea y Hesperioidea (Lamas 2000).

Las mariposas por su específica relación con la flora y su sensibilidad a los cambios ambientales, resultan excelentes indicadores de diversidad y elementos valiosos en los programas de conservación, monitoreo y educación ambiental (Sparrow et al. 1994). Las modificaciones ecológicas, debido a las alteraciones de los hábitats naturales por acción antropogénica, conllevan la necesidad de preservar la biodiversidad, para lo cual resulta indispensable el estudio de la riqueza de especies de una zona determinada (Morrone \& Coscarón 1998).

El desarrollo urbano es una de las actividades humanas que provoca la mayor tasa de extinción de especies nativas (Marzluff 2001) y una de las más importantes amenazas para la biodiversidad (Ricketts \& Imhoff 2003). Como resultado de la urbanización, hay una pérdida 
irreversible de los hábitats naturales, con un consecuente impacto negativo en la biodiversidad local (Pin Koh \& Sodhi 2004).

Así como la reducción de las áreas naturales conduce, en forma inevitable, a la disminución de las poblaciones silvestres, influye también en la pérdida de variabilidad genética y en el aumento de la vulnerabilidad de las especies (Leigh et al. 1993). Blair \& Launer (1997) estudiaron el efecto negativo de la urbanización en la presencia y abundancia de lepidópteros. Los resultados logrados en diferentes investigaciones indican que la riqueza de las mariposas disminuye a medida que el grado de urbanización aumenta (Blair 2001, Brown \& Freitas 2002). La construcción de edificios y carreteras no solamente reduce el área de los hábitats naturales, sino que afecta también la calidad de los hábitats residuales (Hardy \& Dennis 1999).

La diversidad de mariposas en Argentina es alta, debido a la gran variedad de climas, alturas y la amplia disponibilidad de recursos alimenticios. Se dispone de catálogos de la fauna de lepidópteros (Tricio et al. 2002, Canals 2003, Pastrana 2004) y de estudios realizados en las provincias de Buenos Aires y Misiones (Núñez 2007, 2008a, 2008b) y en Corrientes y Chaco (Bar et al. 2005, 2008a, 2008b).

Los objetivos del trabajo fueron conocer la estructura y composición de la comunidad de Hesperioidea y Papilionoidea y analizar su variación estacional en la ciudad de Corrientes.

\section{MATERIALES Y MÉTODOS}

Área de estudio: El área explorada pertenece al Distrito Oriental Húmedo de la Provincia Fitogeográfica Chaqueña (Cabrera \& Willink 1973, Carnevali 1994). La Región Neotropical ocupa la mayor parte de América del Sur, el Dominio Chaqueño, incluido en la región Neotropical se extiende desde el sur de Bolivia, hasta Córdoba, San Luis, llegando por el Este hasta el Norte de Corrientes (Morrone 2001). El clima es subtropical, las lluvias superan los $1500 \mathrm{~mm}$ anuales. La temperatura media anual varía entre 19 y $23^{\circ} \mathrm{C}$. Las temperaturas máximas absolutas oscilan entre 42.5 y $46.5^{\circ} \mathrm{C}$ y las mínimas absolutas entre -1 y $-5^{\circ} \mathrm{C}$ (Cabrera \& Willink 1973).

El estudio se efectuó en la ciudad de Corrientes, donde se concentra el 95\% del total de la población humana del Departamento Capital, provincia de Corrientes (INDEC 2001). Se seleccionaron dos ambientes: un parche de bosque, localizado en el área suburbana a $12 \mathrm{~km}$ del centro de la ciudad, en el Barrio Santa Catalina $\left(27^{\circ} 32^{\prime} 75^{\prime \prime} \mathrm{S}-58^{\circ} 46^{\prime} 30^{\prime \prime} \mathrm{W}\right)$, con una extensión de $51000 \mathrm{~m}^{2}$ y sin perturbaciones antrópicas significativas; y un espacio verde, el Parque Mitre, ubicado en el Barrio Aldana (27²7'39” S - 58 49'42” W), en la zona céntrica de la ciudad, rodeado por una matriz de edificaciones, con una superficie de aproximadamente $44000 \mathrm{~m}^{2}$.

Muestreo entomológico: Los muestreos se llevaron a cabo en verano (enero y febrero), otoño (abril y mayo), invierno (junio y agosto) y primavera (septiembre y octubre), en un período que abarcó desde el 2 de enero al 12 de octubre del 2007. Se realizaron dos muestreos al azar durante cada estación climática, con un esfuerzo de captura de dos personas por día de muestreo, de las 9 a las 13 hrs y de las 14 a las 16hrs.

Las mariposas se registraron visualmente y las especies no identificadas se colectaron mediante redes entomológicas. El material capturado, separado en camas de papel de filtro, se conservó en cajas de plástico rotuladas, hasta su identificación.

Trabajo de laboratorio: Para la identificación taxonómica se utilizaron claves y descripciones de Costa Lima 1945, 1950, Borror et al. 1989, Canals 2003 y Pastrana 2004. Los ejemplares no identificados se enviaron al naturalista Ezequiel Núñez Bustos, integrante de la Fundación de Historia Natural Félix de Azara. Los especímenes se incorporaron a la colección de la Cátedra de Biología de los Artrópodos de la Universidad Nacional del Nordeste (CARTROUNNE), Corrientes, Argentina. 
Análisis de datos: Los índices de diversidad de Shannon-Wiener, equitatividad y de dominancia de Berger-Parker se calcularon con el programa Bio-Dap (Thomas 2000). El índice de similitud cuantitativo de Morisita-Horn y el cualitativo de Jaccard se utilizaron para comparar la composición de los lepidópteros entre las unidades ambientales estudiadas (Magurran 1988). En el análisis estadístico se aplicaron el test de $\chi^{2}$ y el test $t$ a un nivel de significación del 5\% (Sokal \& Rohlf 1995).

\section{RESULTADOS}

El total de lepidópteros registrados en las unidades ambientales Santa Catalina y Parque Mitre, comprendió seis familias, 59 géneros, 75 especies y 1114 individuos, de los cuales 701 fueron registrados en Santa Catalina y 413 en el Parque Mitre (Cuadro 1). Nymphalidae $(n=465)$ fue la familia más abundante y la más diversa identificándose 28 especies. Hesperiidae $(\mathrm{n}=312)$ resultó la segunda familia

CUADRO 1

Lepidoptera de las superfamilias Papilionoidea y Hesperioidea de Corrientes, Argentina 2007

TABLE 1

Lepidoptera (Papilionoidea and Hesperioidea) list from Corrientes, Argentina 2007

\begin{tabular}{|c|c|c|}
\hline & Parque Mitre & Santa Cat \\
\hline \multicolumn{3}{|l|}{ Hesperioidea } \\
\hline \multicolumn{3}{|l|}{ Hesperiidae } \\
\hline \multicolumn{3}{|l|}{ Hesperiinae } \\
\hline \multicolumn{3}{|l|}{ Arita mubevensis (Bell, 1932) } \\
\hline \multicolumn{3}{|l|}{ Calpodes ethlius (Stoll, 1782) } \\
\hline Cymaenes gisca Evans, 1955 & $\mathrm{X}$ & $\mathrm{X}$ \\
\hline Hylephila phyleus (Drury, 1773) & $\mathrm{X}$ & $\mathrm{X}$ \\
\hline Panoquina ocola (Edwards, 1863) & $\mathrm{X}$ & $\mathrm{X}$ \\
\hline \multicolumn{3}{|l|}{ Pyrrhopygopsis socrates socrates (Ménetriés, 1855) } \\
\hline \multicolumn{3}{|l|}{ Pyrginae/Eudamini } \\
\hline \multicolumn{3}{|l|}{ Autochton zarex (Hübner, 1818) } \\
\hline \multicolumn{3}{|l|}{ Celaenorrhinus similis Hayward, 1933} \\
\hline Chioides catillus (Cramer, 1779) & $\mathrm{X}$ & $\mathrm{X}$ \\
\hline Epargyreus barisses argentina Mabille, 1903 & $\mathrm{X}$ & $\mathrm{X}$ \\
\hline Urbanus dorantes (Stoll, 1790) & $\mathrm{X}$ & $\mathrm{X}$ \\
\hline Urbanus doryssus albicuspis (Herrich - Schäffer, 1869) & $\mathrm{X}$ & $\mathrm{X}$ \\
\hline Urbanus procne (Plötz, 1880) & $\mathrm{X}$ & $\mathrm{X}$ \\
\hline Urbanus proteus (Linnaeus, 1758) & $\mathrm{X}$ & $\mathrm{X}$ \\
\hline Urbanus teleus (Hübner, 1821) & $\mathrm{X}$ & $\mathrm{X}$ \\
\hline \multicolumn{3}{|l|}{ Pyrginae/Pyrgini } \\
\hline \multicolumn{3}{|l|}{ Achlyodes mithridates thraso (Hübner, [1807]) } \\
\hline Heliopetes arsalte (Linnaeus, 1758) & $\mathrm{X}$ & $\mathrm{X}$ \\
\hline Helioptes omrina (Butler, 1870) & $\mathrm{X}$ & $\mathrm{X}$ \\
\hline \multicolumn{3}{|l|}{ Nisoniades macarius (Herrich -Schäffer, 1870) } \\
\hline Pyrgus orcus (Stoll, 1780) & $\mathrm{X}$ & $\mathrm{X}$ \\
\hline \multicolumn{3}{|l|}{ Xenophanes tryxus (Stoll, 1780) } \\
\hline \multicolumn{3}{|l|}{ Pyrrhopyginae/Pyrrhopygini } \\
\hline Pyrrhopyge pelota Plötz, 1879 & $\mathrm{X}$ & $\mathrm{X}$ \\
\hline
\end{tabular}


CUADRO 1 (continuación)

Lepidoptera de las superfamilias Papilionoidea y Hesperioidea de Corrientes, Argentina 2007

TABLE 1 (Continued)

Lepidoptera (Papilionoidea and Hesperioidea) list from Corrientes, Argentina 2007

\section{Papilionoidea}

Parque Mitre

Santa Catalina

Lycaenidae

Polyommatinae

Hemiargus hanno (Stoll, 1790)

Leptotes cassius (Cramer, 1775)

X

X

X

$\mathrm{X}$

Theclinae/Eumaeini

Arawacus separata (Lathy, 1926)

X

Calycopis caulonia (Hewitson, 1877)

$\mathrm{X}$

Nymphalidae

Apaturinae

Doxocopa agathina vacuna (Godart, [1824])

Doxocopa kallina (Staudinger, 1886)

Biblidinae/Biblidini

Hamadryas februa februa (Hübner, [1823])

Pyrrhogyra neaerea arge Goose, 1880

Charaxinae/Anaeini

Memphis moruus stheno (Prittwitz, 1865)

Zaretis isidora (Cramer, 1779)

Danainae/Danaini

Danaus erippus (Cramer, 1775)

Danaus gilippus gilippus (Cramer, 1775)

Heliconiinae/Argynnini

Euptoieta hortensia (Blanchard, 1852)

Heliconiinae/Heliconiini

Agraulis vanillae maculosa (Stichel, [1908])

Dryadula phaetusa (Linnaeus, 1758)

Dryas iulia alcionea (Cramer, 1779)

Heliconius erato phyllis (Fabricius, 1775)

Libytheinae

Libytheana carinenta carinenta (Cramer, 1777)

Morphinae/Brassolini

Brassolis sophorae vulpeculus Stichel, 1902

Opsiphanes invirae amplificatus Stichel, 1904

Morphinae/Morphini

Morpho helenor achillides (C. Felder \& R. Felder, 1867)

Nymphalinae/Kallimini

Anartia amathea roeselia (Eschscholtz, 1821)

Anartia jatrophae jatrophae (Linnaeus, 1763)

Junonia genoveva hilaris C. Felder \& R. Felder, 1867

Nymphalinae/Melitaeini

Anthanassa frisia hermas (Hewitson, 1864)

Chlosyne lacinia saundersi (Doubleday, [1847])
$\mathrm{X}$

$\mathrm{X}$

X

X

X

X

X

X

X

X

X

X

X

$\mathrm{X}$

X $\mathrm{X}$

X $\mathrm{X}$

X $\mathrm{X}$

X $\quad X$

X

X $\quad X$

X $\quad$ X

X $\quad$ X

$\mathrm{X} \quad \mathrm{X}$

$\mathrm{X} \quad \mathrm{X}$

$\mathrm{X} \quad \mathrm{X}$

$\mathrm{X} \quad \mathrm{X}$

$\mathrm{X}$ 
CUADRO 1 (continuación)

Lepidoptera de las superfamilias Papilionoidea y Hesperioidea de Corrientes, Argentina 2007

TABLE 1 (Continued)

Lepidoptera (Papilionoidea and Hesperioidea) list from Corrientes, Argentina 2007

\section{Nymphalinae/Nymphalini}

Parque Mitre

Santa Catalina

Vanessa braziliensis (Moore, 1883)

\section{Satyrinae/Satyrini}

Hermeuptychia hermes (Fabricius, 1775)

Paryphthimoides eous (Butler, 1867)

Pharneuptychia phares (Godart, [1824])

X

$\mathrm{X}$

Taygetis laches marginata Staudinger, [1887]

Yphthimoides celmis (Godart, [1824])

Papilionidae

Papilioninae/Graphiini

Mimoides lysithous rurik (Eschscholtz, 1821)

Papilioninae/Papilionini

Heraclides anchisiades capys (Hübner, [1809])

Heraclides thoas brasiliensis (Rothschild \& Jordan, 1906)

Papilioninae/Troidini

Battus polydamas polydamas (Linnaeus, 1758)

Parides anchises nephalion (Godart, 1819)

Parides bunichus perrhebus (Boisduval, 1836)

Parides neophilus eurybates (Gray, [1853])

Pieridae

Coliadinae

Colias lesbia (Fabricius, 1775)

Eurema deva (Doubleday, 1847)

Eurema elathea flavescens (Chavannes, 1850)

Eurema phiale paula (Röber, 1909)

Phoebis neocypris neocypris (Hübner, [1823])

Phoebis philea philea (Linnaeus, 1763)

Phoebis sennae marcellina (Cramer, 1777)

Pyrisitia leuce leuce (Boisduval, 1836)

Pyrisita nise tenella (Boisduval, 1836)

Rhabdodryas trite banksi (Breyer, 1939)

Pierinae/Pierini

Ascia monuste automate (Burmeister, 1878)

Glutophrissa drusilla (Cramer, 1777)

Riodinidae

Riodininae/Riodinini

Caria marsyas Godman, 1903

Melanis aegates cretiplaga (Stichel, 1910)

$\begin{array}{ll}X & X \\ X & X \\ X & X \\ & X \\ X & X\end{array}$

X

$\mathrm{X}$

X

X

$\mathrm{X}$

X

$\mathrm{X}$

$\mathrm{X}$

$\mathrm{X}$

X $\quad X$

X $\mathrm{X}$

X $X$

$\mathrm{X} \quad \mathrm{X}$

X $\quad X$

X $\mathrm{X}$

X $\quad X$

$\mathrm{X} \quad \mathrm{X}$

X $\quad X$

$\mathrm{X}$

X $\quad X$

X $\quad$ X

$\mathrm{X}$

$X$
$X$ 
en abundancia y número de especies $(\mathrm{S}=22)$. En menor proporción se capturaron Pieridae $(n=252)$ con 12 especies, Lycaenidae $(n=46)$ con cuatro especies, Papilionidae $(n=26)$ con siete especies y en Riodinidae ( $\mathrm{n}=13)$ se identificaron dos especies.

Se determinaron tres subfamilias de Hesperiidae: Pyrginae $(n=233)$, Hesperiinae $(n=72)$ y Pyrrhopyginae $(n=7)$. Se identificaron nueve subfamilias de Nymphalidae, de las cuales la mejor representada cuantitativamente fue Nymphalinae $(\mathrm{n}=190)$, seguida de Heliconiinae $(\mathrm{n}=139)$ y Satyrinae $(\mathrm{n}=59)$.

En Pieridae se identificaron Coliadinae $(n=218)$ y Pierinae $(n=34)$. Entre los Lycaenidae se identificó a Polyommatinae $(n=44)$, y a Theclinae $(n=2)$, en Papilionidae y Riodinidae sólo se determinó una subfamilia. En el Parque Mitre no se hallaron las subfamilias Charaxinae ni Libytheinae.

En Santa Catalina se identificaron 58 géneros y 39 en el Parque Mitre. Los géneros más diversos fueron: Urbanus con cinco especies y Eurema, Phoebis y Parides con tres. Heliopetes, Doxocopa, Danaus, Anartia, Heraclides y Pyrisitia con dos especies.

De las 75 especies identificadas, 51 se registraron en las dos áreas de estudio. En Santa Catalina se hallaron 74 especies, de las cuales 27 pertenecen a Nymphalidae y 23 resultaron exclusivas de esta área. En el Parque
Mitre se encontraron 52 especies de las cuales 22 se agrupan entre los Nymphalidae. Se destaca el hallazgo de Hamadryas februa februa, (Biblidinae) no detectada en Santa Catalina.

Cuatro especies de Hesperioidea y 11 de Papilionoidea fueron de aparición única. Las especies raras halladas son: Celaenorrhinus similis, Arawacus separata, Calycopis caulonia, Chlosyne lacinia saundersi y Rhabdodryas trite banksi.

El 92\% (69/75) de las especies se capturaron en primavera y/o verano, 12 se capturaron en las cuatro estaciones muestreadas, y seis especies, todas representadas por un solo ejemplar, se recolectaron exclusivamente en otoño. En Santa Catalina Anartia jatrophae jatrophae fue la especie más abundante en verano, invierno y primavera, mientras que en otoño fue Agraulis vanillae maculosa. En el Parque Mitre, A. jatrophae jatrophae resultó también la especie más abundante en verano e invierno. En otoño A. vanillae maculosa y Dryas iulia alcionea y en primavera Urbanus proteus fueron las especies más numerosas.

En Santa Catalina, el 79\% (555/701) de los ejemplares se detectaron en primavera y verano. En otoño e invierno se constató una notable disminución de la abundancia, recolectándose 114 y 32 individuos respectivamente, representando un $21 \%$ del total (Cuadro 2). En el Parque Mitre $81 \%$ (336/413) de los ejemplares

\section{CUADRO 2}

Abundancia de individuos colectados en las cuatro estaciones climáticas, discriminados según familia y unidad ambiental. Corrientes, Argentina 2007

TABLE 2

Abundance of individuals collected at four climatics seasons, according to family and studied areas. Corrientes, Argentina 2007

\begin{tabular}{lcccccccc}
\multicolumn{1}{c}{} & \multicolumn{3}{c}{ Santa Catalina } & \multicolumn{5}{c}{ Parque Mitre } \\
\multicolumn{1}{c}{ Familias } & Verano & Otoño & Invierno & Primavera & Verano & Otoño & Invierno & Primavera \\
Nymphalidae & 108 & 61 & 13 & 120 & 60 & 30 & 10 & 64 \\
Hesperiidae & 64 & 29 & 10 & 89 & 51 & 15 & 8 & 42 \\
Pieridae & 70 & 18 & 7 & 54 & 52 & 10 & 2 & 41 \\
Lycaenidae & 15 & 2 & 0 & 11 & 8 & 0 & 1 & 9 \\
Papilionidae & 8 & 2 & 2 & 9 & 2 & 0 & 0 & 4 \\
Riodinidae & 4 & 2 & 0 & 3 & 1 & 1 & 0 & 2 \\
Total & 269 & 114 & 32 & 286 & 174 & 56 & 21 & 162
\end{tabular}


se registraron en primavera y verano, en las restantes estaciones la abundancia descendió a 56 ejemplares en otoño y sólo 21 en invierno.

La abundancia y la diversidad, según el índice de Shannon, fueron superiores en Santa Catalina. El índice de dominancia fue similar en ambas unidades de paisaje, y la similitud cualitativa y cuantitativa fue alta (Cuadro 3 ). Se observaron diferencias estadísticas significativas entre las cuatro estaciones climáticas, en cuanto al número de individuos, tanto en Santa Catalina $\left(\chi^{2}=258.6\right.$, g.l. $\left.=3, \mathrm{p}=0.0001\right)$ como en el Parque Mitre $\left(\chi^{2}=169.0\right.$, g.l. $\left.=3, \mathrm{p}=0.0001\right)$. Se observó diferencia estadísticamente significativa entre la diversidad de Santa Catalina y del Parque Mitre ( $\mathrm{t}=6.70, \mathrm{p} \leq 0.05)$.

\section{CUADRO 3}

Diversidad de la lepidopterofauna según sitios de muestreo. Corrientes, Argentina 2007

TABLE 3

Diversity of Lepidoptera according to sampled sites. Corrientes, Capital. Argentina 2007

$\begin{array}{lcc} & \text { Santa Catalina } & \text { Parque Mitre } \\ \text { Abundancia (N) } & 701 & 413 \\ \text { Riqueza especifica (S) } & 74 & 52 \\ \text { Diversidad de Shannon-Wiener (H') } & 3.87 & 3.58 \\ \text { Equitatividad (E) } & 0.90 & 0.90 \\ \text { Berger-Parker Dominance (d) } & 0.080 & 0.085 \\ \text { Índice de Similitud Jaccard } & & 0.68 \\ \text { Índice de Similitud Morisita-Horn } & & 0.93\end{array}$

\section{DISCUSIÓN}

La diversidad de los Lepidoptera colectados en la ciudad de Corrientes podría atribuirse a la heterogeneidad espacial, relacionada con la estructura compleja de la vegetación y a la presencia de numerosas plantas hospedadoras que sirven de alimento a larvas y adultos. Asimismo, Colwell (1994) resalta que una alta diversidad puede ser mantenida en ambientes con perturbación intermedia siempre que en éstos se preserve una elevada heterogeneidad.

En Santa Catalina, a lo largo del muestreo longitudinal, la abundancia de cada una de las familias identificadas fue mayor que la del Parque Mitre. El número de especies de mariposas en ambas áreas es semejante al rango de riqueza, que varió entre 50 y 75 especies, encontrado por Ramírez et al. (2007) en zonas verdes urbanas, si bien el esfuerzo y la superficie muestreada por estos autores fueron mayores.

La comunidad de lepidópteros en el área de estudio se caracteriza por una asociación de familias dominantes como: Nymphalidae,
Hesperiidae, Pieridae, y en menor proporción Lycaenidae, Papilionidae y Riodinidae. Como mencionan Fraija \& Fajardo (2006), todas las familias de Hesperioidea y Papilionoidea citadas para la región Neotropical sobreviven en el área urbana y suburbana.

Se comprobó que Nymphalidae fue la familia dominante en las dos unidades de estudio y en todas las estaciones climáticas, en las cuales $A$. jatrophae jatrophae fue las más abundante. Esta familia resultó también la más diversa, seguida de Hesperiidae, en coincidencia con lo verificado por otros autores (Iserhard \& Romanowski 2004, Núñez 2007, Bar et al. 2008b). Urbanus fue el género más diverso en ambas unidades de muestreo en concordancia con lo constatado por Pinheiro \& Emery (2006), Núñez (2007) y Bar et al. (2008a).

Núñez (2007, 2008a, 2008b) en Misiones y en Buenos Aires, Argentina, halló las seis familias detectadas en Santa Catalina y en el Parque Mitre, resultando Nymphalidae la de mayor número de subfamilias, ocho de las 
cuales coincidieron con las señaladas en esta investigación. Todas las familias y subfamilias halladas en esta investigación, (excepto Pyrrhopyginae entre los Hesperiidae) y 32 especies coinciden con las citadas para Rio Grande do Sul (Iserhard \& Romanowski 2004). Contreras \& Contreras (2008) en el Departamento Ñeembucú, sudoeste de la región oriental del Paraguay, identificaron 12 subfamilias de Nymphalidae, nueve de estas subfamilias y 24 géneros fueron hallados en este estudio.

Aproximadamente en el 50\% (38/75) de las especies se colectaron menos de 10 ejemplares, lo que podría atribuirse a un esfuerzo de muestreo insuficiente. Asimismo, el escaso número de Papilionidae se debe al horario en que se efectuaron las capturas, ya que esta familia incluye especies de hábitos crepusculares (Ramírez et al. 2007).

A. jatrophae jatrophae fue la especie más abundante en el verano, invierno y primavera, y se la capturó durante todo el año en las dos áreas de muestreo, lo que sugiere una superposición de generaciones de la especie investigada. En ambas unidades de muestreo A. vanillae maculosa fue dominante en otoño y primavera, situación similar a lo constatado por Bar et al. (2008b) quienes expresan que A. vanillae maculosa se caracteriza por hallarse principalmente en verano y primavera.

En el Parque Nacional de Restinga de Jurubatiba las especies dominantes de Lepidoptera se presentan durante pocos meses, sin embargo, muchas especies con pocos individuos se mantienen constantes todo el año (Esperanço et al. 2003). En contraposición, en el presente estudio las especies dominantes como A. jatrophae jatrophae se visualizaron todo el año y especies con pocos individuos como Epargyreus barisses argentina no se hallaron en forma constante en el período de exploración.

La temperatura es uno de los principales factores abióticos que influye en la reproducción y el desarrollo de los insectos, aunque en algunos casos sus efectos pueden ser modificados por factores tales como la intensidad lumínica y la presencia de plantas hospedadoras.
Así, en esta contribución, la composición de la lepidoptero-fauna varió según la estación climática y las colectas de mayor densidad se observaron en verano y primavera. Es evidente que las bajas temperaturas del invierno y otoño influyeron en el número de ejemplares capturados.

La similitud de Jaccard entre ambas unidades de paisaje muestra que la comunidad de lepidópteros es semejante en cuanto a su carácter cualitativo, el valor del índice de MorisitaHorn indica una semejanza aún mayor entre las dos áreas de estudio. Sin embargo, la abundancia y riqueza fueron mayores en Santa Catalina, mientras que en el Parque Mitre el número de especies fue menor. La menor diversidad y riqueza específica se deben probablemente a factores externos como el mayor grado de urbanización observado en el área circundante, sin embargo, no debería descartarse la influencia de factores internos, como la menor diversidad de hábitats del Parque Mitre en relación a Santa Catalina. Asimismo, Blair \& Launer (1997) se refieren al incremento de riqueza y diversidad de mariposas en sitios con moderado disturbio y a una disminución de la abundancia en áreas urbanas.

La riqueza específica fue elevada teniendo en cuenta que en cada estación climática sólo se llevaron a cabo dos días de muestreo. El mismo número de especies fue censado en la Reserva Ecológica Costanera Sur, un ambiente silvestre de la ciudad de Buenos Aires (Núñez 2008b). Por otra parte, en Santa Catalina, ambiente que conserva un relicto de bosque nativo, se halló un significativo número de especies. Igualmente Blair (2001) resalta que la riqueza de varios taxones, entre los que se destacan los lepidópteros, tiende a ser mayor en áreas suburbanas. Mientras que Brown \& Freitas (2002) indican que los parques urbanos al incluir cierta variedad de vegetación presentan una alta diversidad de lepidópteros, aunque si el tamaño de los parques es reducido y no existe una heterogeneidad espacial, algunas especies resultan pobremente representadas o ausentes.

Es necesario incrementar el número de muestreos, ampliar los horarios de captura e 
incluir otras técnicas, como por ejemplo las trampas de Van Someren-Rydon con cebo, con el fin de aumentar la probabilidad de detectar especies de tamaños poblacionales bajos, tal como lo expuesto por Summerville et al. (2001).

Se confirmó que el número de especies y la abundancia de Lepidoptera son mayores en áreas naturales y en primavera y verano. El área explorada es rica en especies de lepidópteros diurnos, teniendo en cuenta su importancia en la dinámica ecológica, se recomienda promover en el diseño de los espacios verdes de la ciudad, la inclusión de una mayor diversidad de especies vegetales a efectos de mantener o elevar el número de especies de lepidópteros.

\section{RESUMEN}

El proceso de urbanización representa una de las amenazas más importantes a la biodiversidad. Los lepidópteros son uno de los grupos taxonómicos utilizados como indicadores de la diversidad y calidad del ambiente. El objetivo del presente trabajo es conocer los Lepidoptera (Papilionoidea y Hesperioidea) de la ciudad de Corrientes. Se llevaron a cabo muestreos al azar en un parche de bosque nativo situado en el barrio Santa Catalina y en un área antropizada, el Parque Mitre. Las recolectas se realizaron en las cuatro estaciones climáticas entre enero y octubre de 2007 con redes entomológicas. El total de ejemplares capturados asciende a 1114 , los que se distribuyen en seis familias: Hesperiidae, Lycaenidae, Nymphalidae, Papilionidae, Pieridae y Riodinidae y 18 subfamilias. Se identificaron 59 géneros y 75 especies. Anartia jatrophae jatrophae fue la especie más abundante en ambas unidades. Esta especie junto a Urbanus procne, Phoebis sennae marcellina, Pyrgus orcus y Dryas iulia alcionea se capturaron en todas las estaciones. El mayor número de ejemplares se colectó en las estaciones más cálidas. La abundancia $(\mathrm{n}=701)$, riqueza $(\mathrm{S}=74)$ y diversidad $\left(\mathrm{H}^{\prime}=3.87\right)$ fueron superiores en Santa Catalina. Las unidades exploradas exhiben una elevada riqueza de especies y alta similitud

Palabras clave: lepidópteros diurnos, Hesperioidea, Papilionoidea, riqueza de especies, estacionalidad, parque urbano, área suburbana.

\section{REFERENCIA}

Bar, M.E., M.P. Damborsky, E.B. Oscherov, E. Núñez \& G. Avalos. 2008a. Contribución al conocimiento de los Lepidópteros de la Reserva Provincial Iberá, Corrientes, Argentina. Miscelánea INSUGEO 17: 317-330.
Bar, M.E., E.B. Oscherov, M.P. Damborsky, G. Avalos \& E. Núñez. 2008b. Primer inventario de la fauna de Arthropoda de la Región Chaqueña Húmeda. Miscelánea INSUGEO 17: 331-353.

Bar, M.E., M.P. Damborsky, G. Avalos, E. Monteresino \& E.B. Oscherov. 2005. Fauna de Arthropoda de la Reserva Iberá, Corrientes, Argentina. Miscelánea INSUGEO 14: 293-310.

Blair, R.B. \& A.E. Launer. 1997. Butterfly diversity and human land use: Species assemblages along an urban gradient. Biol. Conservat. 80: 113-125.

Blair, R.B. 2001. Birds and butterflies along urban gradients in two ecoregions of the United States: Is urbanization creating a homogeneous fauna?, p. 33-56. In J.L. Lockwood \& M.L. Mc Kinney (eds.). Biotic Homogenization. Kluwer, Norwell, Massachusetts, EEUU.

Borror, D.J., C.A. Triplehorn \& N.F. Johnson. 1989. An Introduction to the Study of Insects. Saunders, Filadelfia, Pensilvania, EEUU.

Brown, K.S. \& A.V.L. Freitas. 2002. Butterfly communities of urban forest fragments in Campinas, São Paulo, Brazil: structure, instability, environmental correlates, and conservation. J. Insect. Conservat. 6: 217-231.

Cabrera, A.L. \& A. Willink. 1973. Biogeografía de América Latina. Serie de Biología, Monografía No 13 , Washington D.C., EEUU.

Canals, G.R. 2003. Mariposas de Misiones. L.O.L.A., Buenos Aires, Argentina.

Carnevali, R. 1994. Fitogeografía de la Provincia de Corrientes. Gobierno de la Provincia de Corrientes. INTA, Corrientes, Argentina.

Colwell, R.K. 1994. Estimating terrestrial biodiversity through extrapolation, p. 101-118. In D.L. Hawksworth (ed.). The quantification and estimation of organismal biodiversity. Phil. Trans. R. Soc., London, England.

Contreras, A.O. \& J.R. Contreras. 2008. Lista preliminar de especies de la Familia Nymphalidae (Papillonoidae) en el Departamento Neembucú. Sudoeste de la Región Oriental, República del Paraguay. CIENCIAS Revista de la. Universidad de. Maimónides 1: 67-71.

Costa Lima, A. 1945. Insetos do Brasil, $5^{\circ}$ tomo, Lepidópteros. Escola Nacional de Agronomia, Rio de Janeiro, Brasil.

Costa Lima, A. 1950. Insetos do Brasil, $6^{\circ}$ tomo, Lepidópteros Escola Nacional de Agronomia, Rio de Janeiro, Brasil. 
Esperanço, A.P., E.V. Herkenhoff \& R.F. Monteiro. 2003. Distribução de Lepidópteros em dois hábitats do Parque Nacional da Restinga de Jurabatiba (RJ), p. 39-40. In VI Congresso de Ecologia do Brasil, Fortaleza, Brasil.

Fraija, N. \& G.E. Fajardo. 2006. Caracterización de la fauna del orden Lepidoptera (Rhopalocera) en cinco diferentes localidades de los Llanos Orientales colombianos. Acta biol. Colomb. 11: 55-68.

Hardy, P.B. \& R.L.H. Dennis. 1999. The impact of urban development on butterflies within a city region. Biodivers. Conserv. 8: 1261-1279.

INDEC. 2001. Instituto Nacional de Estadísticas y Censos. Censo 2001, Corrientes, Argentina. (Consultado 10 marzo 2010, www.indec.mecon.ar).

Iserhard, C.A. \& H.P. Romanowski. 2004. Lista de espécies de borboletas (Lepidoptera, Papilionoidea e Hesperioidea) da região do vale do rio Maquiné, Rio Grande do Sul, Brasil. Rev. Bras. Zool. 21: 649-662.

Lamas, G. 2000. Estado actual del conocimiento de la sistemática de los lepidópteros, con especial referencia a la Región Neotropical, p. 253-260. In M.F. Piera, J.J. Morrone \& A. Melic (eds.). Hacia un Proyecto CYTED para el Inventario y Estimación de la Diversidad Entomológica en Iberoamérica: PriBES 2000 (Monografía Tercer Milenio). Sociedad Entomológica Aragonesa, Zaragoza, España.

Leigh Jr. E.G., S.J. Wright, E.A. Herre \& F.E. Putz. 1993 The decline of tree diversity on newly isolated tropical islands: A test of the hypothesis and some implications. Evol. Ecol. 7: 76-102.

Magurran, A. 1988. Ecological diversity and its measurement. Cambridge, Cambridge, England.

Marzluff, J.M. 2001. Documenting the Apocalypse. Conservat. Biol. 14: 1219-1220.

Morrone, J.J. \& S. Coscarón. 1998. Introducción a la Biodiversidad de Artrópodos Argentinos. Una perspectiva biotaxonómica. Sur, La Plata, Argentina.

Morrone, J.J. 2001. Biogeografía de América Latina y el Caribe. Manuales \& Tesis de la SEA, CYTED, ORCYT - UNESCO, Zaragoza, España.

Núñez, E.O. 2007. Biogeografía de los Rhopalocera de la isla Martín García, provincia de Buenos Aires, Argentina (Lepidoptera: Papilionoidea y Hesperioidea). SHILAP Rev. Lepidopt. 35: 289-309.

Núñez, E.O. 2008a. Diversidad de mariposas diurnas en la Reserva Privada Yacutinga, provincia de Misiones,
Argentina (Lepidoptera: Hesperioidea y Papilionoidea). Trop. Lepid. Res. 18: 78-87.

Núñez, E.O. 2008b. Las especies urbanas de Rhopalocera de la Reserva Ecológica Costanera Sur, Ciudad de Buenos Aires, Argentina (Lepidoptera: Hesperioidea y Papilionoidea). SHILAP Rev. Lepidop. 36: 435-447.

Pastrana, J.A. 2004. Los Lepidópteros Argentinos: sus plantas hospedadoras y otros sustratos alimenticios. Sociedad Entomológica Argentina, San Miguel de Tucumán, Argentina.

Esperanço, A.P, E. Vieira Herkenhoff \& R. Ferreira Monteiro. 2003. Distribução de Lepidópteros em dois hábitats do Parque Nacional da Restinga de Jurabatiba (RJ), p. 39-40. In VI Congresso de Ecologia do Brasil, Fortaleza, Brasil.

Pin Koh, L. \& N.S. Sodhi. 2004. Importance of reserves, fragments, and parks for butterfly conservation in a tropical urban landscape. Ecol. Appl. 14: 1695-1708.

Pinheiro, C.E.G. \& E.O. Emery. 2006. As borboletas (Lepidoptera: Papilionoidea e Hesperioidea) da Área de Proteção Ambiental do Gama e Cabeça de Veado (Distrito Federal, Brasil). Biota Neotrop. 6: 1-15.

Ramírez, L., P. Chacón \& L.M. Constantino. 2007. Diversidad de mariposas diurnas (Lepidoptera: Papilionoidea y Hesperioidea) en Santiago de Cali, Valle del Cauca, Colombia. Rev. Colomb. Entomol. 33: 54-63.

Ricketts, T. \& M. Imhoff. 2003. Biodiversity, urban areas, and agriculture: locating priority ecoregions for conservation. Conserv. Ecol. 8: 1. (Consultado 12 marzo 2010, http://www.consecol.org/vol8/iss2/art1).

Sokal, R.R. \& F.J. Rohlf. 1995. Biometry. W.H. Freeman, Nueva York, Nueva York, EEUU.

Sparrow, H.R., T.D. Sisk, P.R. Ehrlich \& D.D. Murphy. 1994. Techniques and guidelines for monitoring Neotropical butterflies. Conservat. Biol. 8: 800-809.

Summerville, K.S., E.H. Metzler \& T.O. Crist. 2001. Diversity of Lepidoptera in Ohio forests at local and regional scales: how heterogeneous is the fauna? Ann. Entomol. Soc. Am. 94: 583-591.

Thomas, G.M. 2000. Bio- Dap. A biodiversity analysis Packaged (Consultado 1 mayo 2009, http://nhsbig. inhs.uiuc.edu/www/populations.html).

Tricio, A.E., C.I. Fernández \& P.M. Morawichi. 2002. Mariposas de Misiones. Guía para la observación e identificación. MG Grupo Creativo, Buenos Aires, Argentina. 